\title{
Use of Ventilation-Perfusion Single-Photon Emission Computed Tomography to Select the Target Lobe for Endobronchial Valve Lung Volume Reduction
}

\author{
Vanessa Sze Theen Tee ${ }^{a, b}$ Phan Nguyen ${ }^{a, b}$ Hubertus Jersmann ${ }^{a, b}$ \\ Dion Grosser ${ }^{c}$ Benjamin Crouch ${ }^{d}$ Brett Lorraine ${ }^{e}$ Chong Ghee Chew ${ }^{d}$ \\ aschool of Medicine, Faculty of Health and Medical Sciences, The University of Adelaide, Adelaide, SA, Australia; \\ ${ }^{b}$ Department of Thoracic Medicine, Royal Adelaide Hospital, Adelaide, SA, Australia; ${ }^{C}$ ChestCare, Adelaide, SA, \\ Australia; ${ }^{d}$ Department of Nuclear Medicine, Royal Adelaide Hospital, Adelaide, SA, Australia; ' $S A$ Medical Imaging, \\ Royal Adelaide Hospital, Adelaide, SA, Australia
}

\section{Keywords}

Bronchoscopic lung volume reduction · Chronic obstructive pulmonary disease $\cdot$ Emphysema $\cdot$ Endobronchial valves . Bronchoscopy $\cdot$ Ventilation-perfusion scan $\cdot$ Single-photon emission computed tomography · Scintigraphy

\footnotetext{
Abstract

Background: Quantitative planar ventilation-perfusion (VQ) has a complementary role in target lobe selection for endobronchial valve lung volume reduction (EBV-LVR), especially in homogenous disease. We investigated a novel method of lung lobar quantitation using VQ single-photon emission computed tomography (SPECT) with computed tomography (CT) to generate a parameter called the ventilation-perfusion differential index (VQDI). Aim: The aim of this study was to validate $V Q D I$ as a parameter for target lobe selection in EBV-LVR against the gold standard test of quantitative computed tomography (qCT). Methods: This study was a prospective, multi-centre, single-blinded, observational study of EBV-LVR patients. Baseline and 3-month post inter-
}

vention VQ SPECT and qCT were performed. The target lobe was chosen using $\mathrm{qCT}$ and planar VQ report $\left(\mathrm{CT}_{\mathrm{TL}}\right)$ whilst blinded to VQDI. Post EBV-LVR, our nuclear physician, blinded to $C_{T}$, selected a target lobe using deidentified VQDI $\left(\mathrm{VQDI}_{\mathrm{TL}}\right)$. Inter-rater agreement between $\mathrm{CT}_{\mathrm{TL}}$ and $\mathrm{VQDI}_{\mathrm{TL}}$ was calculated by Kappa statistic. Treatment outcomes were analysed with a linear mixed-effects model. Results: There was a high concordance between $\mathrm{CT}_{\mathrm{TL}}$ and $\mathrm{VQDI}_{\mathrm{TL}}$ in 16 patients $(89 \%$, Kappa statistic $=0.85)$. Post EBV-LVR, our subjects showed significant changes in FEV1 (mean difference $[\mathrm{MD}]+150 \mathrm{~mL}, p<0.001$ ), target lobe volume reduction (MD $-973 \mathrm{~mL}, p<0.001)$, residual volume (MD $-800 \mathrm{~mL}, p<$ 0.001 ), and St. George's Respiratory Questionnaire score (MD $-11, p=0.001)$. Improvements in 6-minute walk distances did not reach statistical significance. Conclusion: In this study of treatment responders, EBV-LVR target lobe selection using VQDI concurs with $\mathrm{qCT}$ and thus supports its value for this purpose. It complements qCT and may potentially be of synergistic value especially in homogenous emphysema.

(c) 2021 The Author(s).

Published by S. Karger AG, Basel karger@karger.com www.karger.com/res

Karger $\stackrel{\text { ' }}{5}$

BOPEN ACCESS
(C) 2021 The Author(s)

Published by S. Karger AG, Basel

This is an Open Access article licensed under the Creative Commons Attribution-NonCommercial-4.0 International License (CC BY-NC) (http://www.karger.com/Services/OpenAccessLicense), applicable to the online version of the article only. Usage and distribution for commercial purposes requires written permission. 


\section{Introduction}

Selecting the target lobe for endobronchial valve lung volume reduction (EBV-LVR) in severe emphysema can be challenging especially in patients with homogenous disease. Currently quantitative computed tomography (qCT) is the gold standard imaging to guide target lobe choice. qCT provides analysis of the lobar volumes, emphysema ratios, and fissure integrity. Homogenous emphysema is defined as a $<15 \%$ difference in emphysema ratio between the target lobe and the remaining ipsilateral lobe [1-4].

Planar perfusion scintigraphy has a complementary role in target lobe selection especially in homogenous disease [4-6] where the qCT is unable to identify an obvious target. The target lobe in the IMPACT study by Valipour et al. [4] was chosen based on the highest emphysema ratio, absence of collateral ventilation, and the lowest perfusion on planar perfusion scintigraphy. The commonly used current method involves 2-dimensional quantitation of radioactivity in the anterior and posterior projections and segmenting the lungs into 6 symmetrical rectangular zones. A major limitation of this method is the lack of correlation with actual lobar anatomy, particularly in the setting of advanced emphysema.

With the advent of hybrid gamma cameras with CT, single-photon emission computed tomography (SPECT)/ $\mathrm{CT}$ is now a frequently used method of 3-dimensional imaging in nuclear medicine, including for ventilationperfusion (VQ) scans. Fusion of SPECT with CT allows for accurate qualitative evaluation of the physiological data of SPECT with the anatomical detail of CT. Quantitation with SPECT/CT however is not often performed due to associated technical complexities.

VQ SPECT/CT lobar quantitation programs are now commercially available from gamma camera vendors. These programs are capable of determining the percentage radioactivity of each lobe for the VQ SPECT scans. At our institution, we have independently developed an interactive data language (IDL)-based program of lobar quantitation of differential $V$ and Q. This program, which we have termed the Royal Adelaide Hospital Ventilation Perfusion SPECT/CT Quantitation (RAHVQSQ), is capable of analysing data acquired as a SPECT with CT in a singular hybrid camera or with 2 separate datasets consistent with the SPECT from a gamma camera and a chest $\mathrm{CT}$ from another scanner. We have also devised a unique method of deriving an index from the percentages of $\mathrm{V}$ and $\mathrm{Q}$ radioactivity, and the percentage volume of each lobe. We have termed this index the ventilation perfusion differential index (VQDI).

Use of VQ SPECT to Select Target Lobe for EBV-LVR
We suspect the VQDI reflects the gas exchange capacity and thus has potential value of determining the degree of emphysema of each lobe. It could potentially provide the physiological equivalent of the emphysema ratio of $\mathrm{qCT}$. With the exception of fissure integrity, all of the parameters needed for target lobe selection in EBV-LVR provided by qCT can be derived using RAHVQSQ.

Thus, the primary aim of our study was to validate that VQDI is a parameter of value for target lobe selection in EBV-LVR by evaluating the concordance with the current gold standard imaging test of qCT. Our secondary aim was to evaluate the effect of EBV-LVR on the VQDI of the treated and untreated lobes.

\section{Material and Methods}

\section{Study Design}

This study was a prospective, multi-centre, single blinded, observational study. This study was approved by the Royal Adelaide Hospital Human Research Ethics and Central Adelaide Local Health Network Governance (HREC/16/RAH/237 R20160624) and the Calvary Health Care Adelaide Human Research and Ethics Committee (16-CHREC-E007).

\section{Study Subjects}

The recruited participants provided written informed consent and permission to publish. The participants were patients with severe emphysema who remained symptomatic despite maximum medical therapy and were referred for LVR. The inclusion criteria included an age of 40-80 years, diagnosis of emphysema including homogenous emphysema, a forced expiratory volume in $1 \mathrm{~s}$ (FEV1) of $15-50 \%$ of the predicted value, a total lung capacity of $>100 \%$ of the predicted value, a residual volume (RV) of over $150 \%$ of the predicted value, and post-rehabilitation 6-minute walk distance (6MWD) of at least $140 \mathrm{~m}$. The exclusion criteria included corrected carbon monoxide diffusing capacity (DLCOcorr) of $<20 \%$ of the predicted value, hypercapnoea with a partial pressure of carbon dioxide of over $50 \mathrm{~mm} \mathrm{Hg}$, hypoxaemia with partial pressure of oxygen $<45 \mathrm{~mm} \mathrm{Hg}$ while breathing ambient air, giant bullae taking up $>30 \%$ lung volume of target lobe, $\alpha 1$-antitrypsin deficiency, previous thoracotomy on target side, excessive sputum of $>4$ tablespoons per day), uncontrolled severe pulmonary hypertension (pulmonary artery pressure $>50 \mathrm{~mm} \mathrm{Hg}$ ), active infection, unstable cardiac disease, lung nodule that requires further workup to exclude malignancy and contraindications to VQ scan like egg allergy and pregnancy or potential pregnancy. All our subjects were screened at baseline with no co-morbidities as outlined in the exclusion criteria. These inclusion and exclusion criteria were based on publications from Herth et al. [7], Kloosters et al. [2], and Valipour et al. [4].

\section{Methods}

Baseline

At enrolment, all participants had qCT chest and standard VQ SPECT along with completed St. George's Respiratory Questionnaire (SGRQ). They also underwent standard physiological testing 
Fig. 1. The operator rotates the image to the desired angle, specifies the target region by annotating a closed curve around the target region which is projected through the whole image. The CT window is adjustable to display the pulmonary vasculature thus accentuating the fissures.

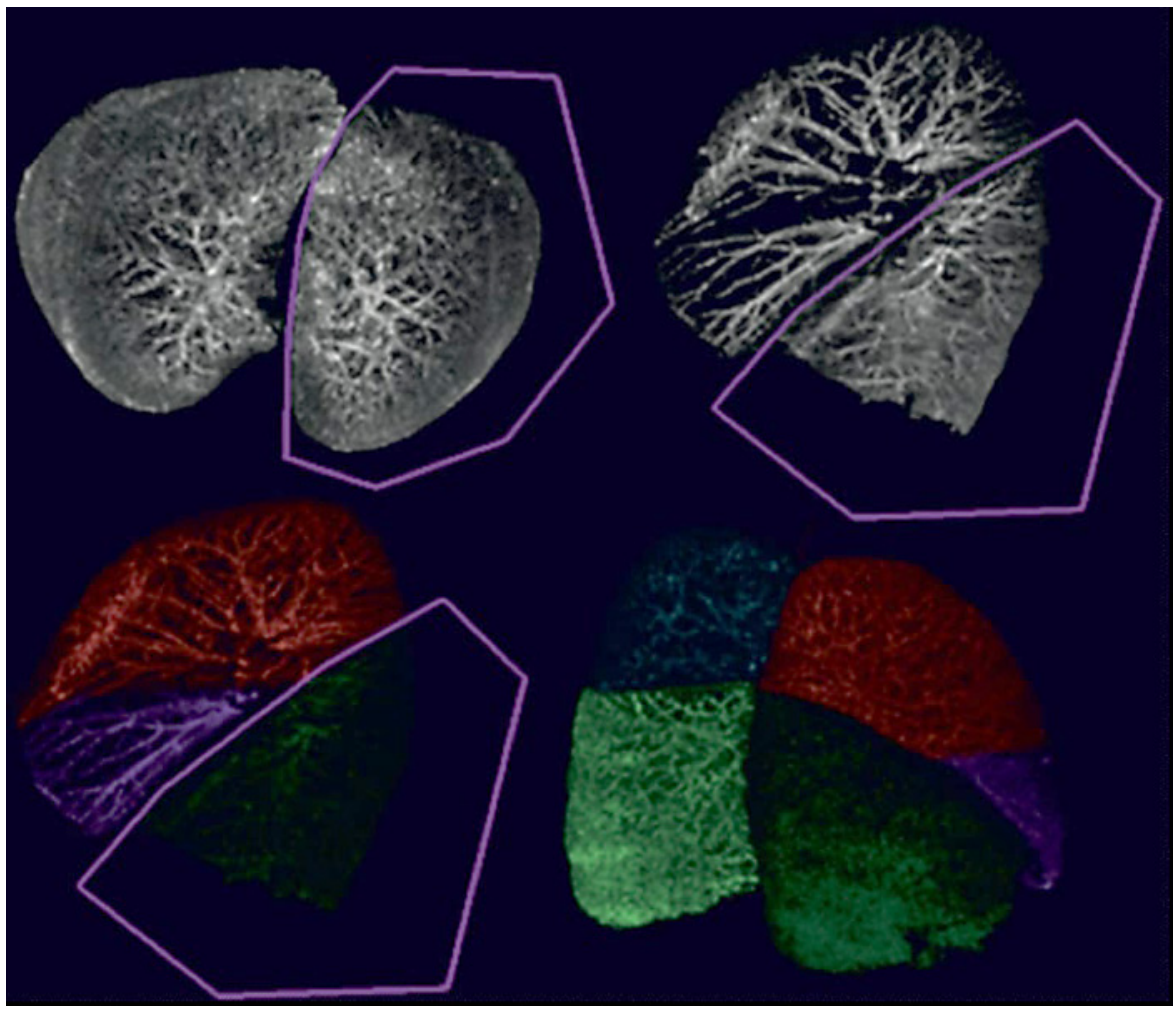

as per the standard protocol, including spirometry, gas transfer, static lung volumes, and 6-minute walk test (6MWT).

\section{Imaging}

The participants had their imaging in various centres depending on the point of recruitment. Most of the CTs were performed on a Siemens Somatom Definition AS 128 detectors (Siemens Healthcare) with acquisition parameters of $120 \mathrm{Kv} 85 \mathrm{mAs} 100$ DLP 200-300 mGy cm CTDi 5-10 mGy, acquired both in inspiration and expiration The quantitative analysis was performed on the Philips IntelliSpace Portal version 10.1 (Philips Healthcare). The CTs were formally reviewed by our thoracic radiologist who determined fissure integrity.

All of the VQ scans were performed on Hawkeye 4 (GE Healthcare). Standard protocol was applied with $40-50 \mathrm{MBq}{ }^{99 \mathrm{~m}} \mathrm{Tc}$ Technegas for the ventilation scan and $200-250 \mathrm{MBq}{ }^{99 \mathrm{~m}} \mathrm{Tc}$-labelled macro aggregated albumin for the perfusion scan. Planar scans ( 3 min acquisition, $256 \times 256$ matrix) were acquired for both studies in the anterior and posterior projections, and segmental quantitation in 6 symmetrical rectangular zones was derived using geometric mean. SPECT scans were acquired for both studies (Matrix 128 $\times 128$; rotation: 3.0 , zoom 1; ventilation $13 \mathrm{~s} / \mathrm{step}$, perfusion $8 \mathrm{~s} /$ step; 120 views; Butterworth filter, threshold 0.48 , power 10 and OSEM 2 iterations 10 subsets). If a low-energy CT was used, the acquisition parameters were $2.5 \mathrm{~mA}, 140 \mathrm{kV}$, DLP $125 \mathrm{mGy} \mathrm{cm}$.

The SPECT and the diagnostic CT datasets were then used for lobar segmentation in the RAHVQSQ program, which is IDLbased version 6.3 software. In this program, the CT is displayed as a 3-dimensional map which can be freely rotated by the operator who determines the positions of the fissures by manual annotation (shown in Fig. 1). The program allows the free manipulation of the windowing of the CT to assist fissure identification. Our nuclear physician (CC) was the sole operator of this program for all studies. Once the fissures are localized, the program then fuses the CT map with the V and Q SPECTs and determines the percentage radioactivity of each scintigraphic study for each lobe. The results are displayed as a spreadsheet, and an example is depicted in Table 1. From the differential percentages of $\mathrm{V}$ and $\mathrm{Q}$ and volume of each lobe, the VQDI was determined.

\section{Target Lobe Selection}

The target lobe was selected at a multi-disciplinary team meeting attended by interventional pulmonologists, thoracic radiologist, nuclear physician, and occasionally thoracic surgeons. The panel made target lobe recommendations based on $\mathrm{CT}\left(\mathrm{CT}_{\mathrm{TL}}\right)$ using the following criteria, including fissure integrity as assessed by a thoracic radiologist and Chartis assessment to ensure absence of collateral ventilation, highest emphysema destruction ratio (percentage of voxels $<950 \mathrm{HU}$ ), high target lobe volume, and emphysema heterogeneity - heterogeneous defined as absolute difference of $15 \%$ or greater in emphysema destruction ratio. Where the qCT showed homogenous emphysema (emphysema destruction ratio $<15 \%$ ), the target lobe was chosen on the basis of the planar perfusion scintigraphy results, in conjunction with other qCT parameters including the fissure integrity and lobar volume. The VQDI results were blinded to the panel (with the exception of the nuclear physician who generated the index and thus did not participate in the panel recommendations) and proceduralists during target lobe selection. 
Table 1. An example of results of RAHVQSQ analysis of VQ SPECT/CT

\begin{tabular}{lllllll}
\hline & $(\mathrm{A})$ Ventilation, $\%$ & $(\mathrm{~B})$ Perfusion, $\%$ & $(\mathrm{C})$ Volume, $\%$ & (D) Ventilation/volume & (E) Perfusion/volume & (F) VQDI \\
\hline LUL & 30.4 & 29.1 & 21.1 & 1.44 & 1.38 & 1.99 \\
LLL & 10.0 & 8.00 & 33.0 & 0.30 & 0.24 & 1.76 \\
RUL & 26.8 & 30.9 & 17.5 & 1.53 & 0.87 & 2.70 \\
RML & 5.70 & 6.00 & 6.90 & 0.83 & 1.21 \\
RLL & 27.1 & 26.1 & 21.5 & 1.26 & 0.72 \\
\hline
\end{tabular}

A: Percentage radioactivity in each lobe for the ventilation scan. B: Percentage radioactivity in each lobe for the perfusion scan. C: Percentage volume in each lobe from the 3D CT, map. $\mathrm{D}=\mathrm{A} / \mathrm{C} . \mathrm{E}=\mathrm{B} / \mathrm{C}$. F = VQDI = D $\times$ E. LUL, left upper lobe; LLL, left lower lobe; RUL, right upper lobe; RML, right middle lobe; RLL, right lower lobe; VQDI, ventilation-perfusion differential index; SPECT, singlephoton emission computed tomography; RAHVQSQ, Royal Adelaide Hospital ventilation perfusion SPECT/CT quantitation.

Post EBV-LVR procedure, the nuclear physician selected the target lobe based on de identified VQDI results $\left(\mathrm{VQDI}_{\mathrm{TL}}\right)$ and fissure integrity information from qCT (as the RAHVQSQ cannot determine fissure integrity). He was blinded to all other data including the panel recommendations and treatment lobe. A target lobe was selected according to the lobe with the lowest or next lowest VQDI, heterogeneous emphysema defined by the substantial difference in VQDI between the target and the ipsilateral lobes, and the lobe with the largest volume as determined in the CT map (see column C in Table 1). A threshold difference in VQDI between the target and the ipsilateral lobe to define heterogeneous emphysema was not determined. If there were to be a choice between 2 equipotential target lobes, the one with the larger difference in target versus ipsilateral lobe VQDI was preferred. The planar VQ quantitation results were not used.

\section{Procedure}

All participants underwent bronchoscopy placement of Zephyr EBV under general anaesthesia. Chartis assessments were performed in all participants to ensure the absence of collateral ventilation prior to EBV placement. Participants with collateral ventilation positive on Chartis were then excluded from the study.

Follow-Up

All participants were followed up to 6 months in this study, in addition to their usual follow-up with their treating physician. At 3 months, qCT scan was performed to determine the target lobe volume reduction and to assess for lobar occlusion. Repeat bronchoscopy and valve replacement were performed if atelectasis was not achieved. VQ SPECT scan was repeated at 3 months from baseline or after the repeat procedure in some participants. The lung function test, 6MWT, and SGRQ were collected at 3- and 6-month follow-up.

\section{Primary and Secondary Outcomes}

The primary outcome was to assess the concordance of the target lobe selected based on the gold standard test, $\mathrm{qCT}\left(\mathrm{CT}_{\mathrm{TL}}\right)$, and $\mathrm{VQDI}_{\mathrm{TL}}$. The secondary outcomes were the changes of VQDI in the treated lobe and the ipsilateral untreated lobe at 3 months and to evaluate the association between the changes in DLCOcorr and VQDI.

Use of VQ SPECT to Select Target Lobe for EBV-LVR

\section{Statistical Analysis}

The sample size was calculated based on the precision of the estimate, using Cohen's kappa. A sample size of 12 is required to allow a 95\% CI in the substantial or almost perfect range (lower $95 \%$ CI for kappa $\geq 0.65$ ). So in 95 out of 100 cases, Cohen's Kappa will fall between 0.65 and 1.00 which is substantial to almost perfect reliability. Statistical software used was R Project for Statistical Computing 3.4.3. The primary outcome was calculated by Kappa statistic. The effect of endobronchial valves on various measurements was assessed by comparing baseline and post-procedure values ( 3 and 6 months). The analysis was done as per protocol by using linear mixed-effects models to adjust for repeated measurement over time to avoid erroneous standard errors and $p$ values due to the same patients being measured 2-3 times (correlation of outcomes within patients). Linear regression models were used to investigate the association between DLCO and VQDI. The statistical software used in the secondary outcomes analysis was SAS 9.4 (SAS Institute Inc., Cary, NC, USA).

\section{Results}

\section{Demographics}

Between November 2016 and May 2018, 25 participants were screened for study eligibility; 4 were excluded due to positive collateral ventilation on Chartis and 3 by way of infection by Burkholderia cepacia, Mycobacterium avium complex, and Pseudomonas aeruginosa. A total of 18 participants were recruited from 3 major centres Royal Adelaide Hospital, The Queen Elizabeth Hospital, and Calvary Wakefield Hospital. Seventeen participants were followed up at 3 months and 15 participants at 6 months. One participant had a fall and sustained a neck of femur fracture, which led to a decline in his physical fitness, requiring nursing home placement and causing him to be lost to follow-up for reasons not related to his original airway disease. Another participant had an unex- 
Table 2. Baseline demographics and clinical characteristics

\begin{tabular}{|c|c|}
\hline Baseline characteristic, $n=18$ & Mean \pm SD \\
\hline Age, years & $65.4 \pm 7.60$ \\
\hline Female sex, $n(\%)$ & $10(55.6)$ \\
\hline $\mathrm{BMI}, \mathrm{kg} / \mathrm{m}^{2}$ & $25.2 \pm 5.00$ \\
\hline Post-BD FEV1, L & $0.85 \pm 0.27$ \\
\hline Post-BD FEV1, \% predicted & $32.3 \pm 7.95$ \\
\hline Post-BD FVC, L & $2.39 \pm 0.60$ \\
\hline Post-BD FVC, \% predicted & $69.7 \pm 12.7$ \\
\hline $\mathrm{RV}, \mathrm{L}$ & $4.19 \pm 0.82$ \\
\hline $\mathrm{RV}, \%$ predicted & $200 \pm 37.1$ \\
\hline TLC, L & $6.72 \pm 1.11$ \\
\hline TLC, \% predicted & $125 \pm 18.5$ \\
\hline DLCOcorr, \% predicted & $38.5 \pm 13.6$ \\
\hline $6 \mathrm{MWD}, \mathrm{m}$ & $336 \pm 113$ \\
\hline SGRQ, total point & $65.1 \pm 11.0$ \\
\hline Target lobe volume, $\mathrm{mL}$ & $1,511 \pm 359$ \\
\hline Target-lobe emphysema ratio voxels below $-950 \mathrm{HU}, \% *$ & $32.1 \pm 12.5$ \\
\hline \multicolumn{2}{|l|}{ Emphysema distribution, $n(\%)^{\wedge}$} \\
\hline Heterogeneous & $10(56)$ \\
\hline Homogeneous & $8(44)$ \\
\hline \multicolumn{2}{|c|}{$\begin{array}{l}\text { BMI, body mass index; post-BD, post-bronchodilator; FEV1, force expiratory volume in } 1 \mathrm{~s} \text {; FVC, force vital } \\
\text { capacity; RV, residual volume; TLC, total lung capacity; DLCOcorr, Diffusing capacity of the lungs for carbon } \\
\text { monoxide corrected; SGRQ, St. George's respiratory questionnaire; HU, Hounsfield units; CT, computed } \\
\text { tomography; } 6 \mathrm{MWD}, 6 \text {-min walk distance. }{ }^{*} \text { Emphysema destruction ratio was assessed as the percentage of } \\
\text { voxels of less than }-950 \mathrm{HU} \text { on CT. ^ } \text { Heterogeneous defined as the difference in the emphysema ratio between } \\
\text { the target and the ipsilateral lobe was } 15 \% \text { or greater. }\end{array}$} \\
\hline
\end{tabular}

pected exacerbation of COPD from parainfluenza a few months post procedure despite lobar atelectasis. She was transferred to a nursing home and missed follow-up at 6 months due to worsening chronic pain from an abdominal hernia and worsening of her anxiety post hospitalization which was not related to her respiratory disease and could not be predicted at the time of screening. Another participant had the Zephyr valves removed before 6-month follow-up. This participant had recurrent exacerbations without pneumonia post procedure and had bronchoscopies showing mucous plugging within the valves with evidence of Aspergillus on the washing. A clinical decision was made to remove the valves as the quality of life score did not improve following a short course of anti-fungal treatment.

The mean age was 65 years with over half being female (55.6\%); mean FEV1 $32 \pm 8 \%$ was predicted, consistent with very severe emphysema, and a mean RV of $200 \pm$ $37 \%$ was predicted, consistent with severe gas trapping. There were 8 (44\%) participants who had homogenous emphysema distribution (shown in Table 2).

\section{Primary Outcome}

There was high agreement between $\mathrm{CT}_{\mathrm{TL}}$ and $\mathrm{VQDI}_{\mathrm{TL}}$ lobe. A total of 16 out of 18 target lobes were concordant, with a concordance rate of $89 \%($ Kappa $=0.85)$ Table 3. In one of the discordant cases, case A (shown in Tables 4 -6) qCT indicated homogenous emphysema and only the left lung oblique fissure was intact. Although the left lower lobe had the higher volume, the left upper lobe was chosen based on planar VQ results, with the left upper zone showing a lower perfusion (9 vs. 14\%). Based on VQDI, there was higher heterogeneity between the left upper lobe and the left lower lobe than emphysema ratio from qCT, thus, in retrospect, justifying treating the left lung. However, the left lower lobe was the preferred target lobe with RAHVQSQ analysis because it had a lower VQDI (0.69 vs. 0.91). Despite achieving complete left upper lobar occlusion, this subject had no sustained treatment response beyond the 3 months with a fall in FEV1 and rise in RV. The DLCOcorr dropped at 6 months after initial improvement at 3 months and his symptoms worsened. Further retrospective review also showed that the destruction score was not particularly high on $\mathrm{qCT}$, and 
Table 3. A cross tabulation of target lobe selection based on CT and VQDI

\begin{tabular}{lllllll}
\hline \multirow{2}{*}{ CT target lobe } & \multicolumn{2}{l}{ VQDI target lobe } & \multicolumn{2}{c}{ Total } \\
\cline { 2 - 6 } & LLL & LUL & RUL & RUL + RML & RLL \\
\hline LLL & 3 & 0 & 0 & 0 & 0 & 3 \\
LUL & $1^{*}$ & 6 & 0 & 0 & 0 & 7 \\
RUL & $1^{\#}$ & 0 & 4 & 0 & 0 & 5 \\
RUL + RML & 0 & 0 & 0 & 3 & 0 & 0 \\
RLL & 0 & 0 & 0 & 0 & 0 & 18 \\
Total & 5 & 6 & 4 & 3 & 0 & 0 \\
\hline
\end{tabular}

LLL, left lower lobe; LUL, left upper lobe; RUL, right upper lobe; RML, right middle lobe; RLL, right lower lobe; CT, computed tomography. Grey shading represents concordant cases. ${ }^{*}$ Discordant case A. ${ }^{\text {\#Discordant }}$ case B.

Table 4. qCT results of discordant case A

\begin{tabular}{lcclc}
\hline & Total volume & Emphysema ratio, \% $(-950 \mathrm{HU})$ & Planar perfusion, \% (geometric mean analysis*) \\
\hline RUL & 1,287 & 8.1 & RUZ & 13 \\
RML & 609 & 6 & RMZ & 29 \\
RLL & 1,925 & 14.4 & RLZ & 18 \\
LUL & 1,367 & 15.9 & LUZ & 9 \\
& & & LMZ & 17 \\
LLL & 1,967 & 15.5 & LLZ & 14 \\
\hline
\end{tabular}

The LUL was chosen as target lobe according to qCT and planar perfusion results. RUL, right upper lobe; RML, right middle lobe; RLL, right lower lobe; LUL, left upper lobe; LLL, left lower lobe; VQDI, ventilationperfusion differential index; qCT, quantitative computed tomography; HU, Hounsfield units. * Planar perfusion geometric mean analysis in 6 equal zones - RUZ, right upper zone; RMZ, right middle zone; RLZ, right lower zone; LUZ, left upper zone; LLZ, left lower zone. The left middle zone result was excluded in this table.

Table 5. RAHVQSQ analysis of discordant case A

\begin{tabular}{lllll}
\hline & Volume, \% & Ventilation, \% & Perfusion, \% & VQDI \\
\hline RUL & 20.2 & 29.3 & 24.9 & 1.79 \\
RML & 8.20 & 9.44 & 12.4 & 1.74 \\
RLL & 25.0 & 18.0 & 23.6 & 0.68 \\
LUL & 19.7 & 20.3 & 17.3 & 0.91 \\
LLL & 27.0 & 22.9 & 21.9 & 0.69 \\
\hline
\end{tabular}

The LLL was chosen as the target lobe which had lower VQDI than the LUL. RUL, right upper lobe; RML, right middle lobe; RLL, right lower lobe; LUL, left upper lobe; LLL, left lower lobe; VQDI, ventilation-perfusion differential index; RAHVQSQ, Royal Adelaide Hospital ventilation perfusion SPECT/CT quantitation.
Table 6. Clinical outcomes of the subject with the discordant case A

\begin{tabular}{lccl}
\hline Clinical outcome & Baseline & 3 months & 6 months \\
\hline FEV1, L (\% pred) & $0.85(29)$ & $1.09(39)$ & $0.83(29)$ \\
RV, L (\% pred) & $4.47(196)$ & $3.33(144)$ & $5.12(223)$ \\
DLCOcorr, \% pred & 52 & 58 & 48 \\
TLV, mL & 1,367 & 0 & na \\
6MWD, m & 330 & 220 & na \\
SGRQ, total point & 66.7 & 56.4 & 70.9 \\
\hline
\end{tabular}

FEV1, force expiratory volume in $1 \mathrm{~s}$; FVC, force vital capacity; $\mathrm{RV}$, residual volume; DLCOcorr, diffusing capacity of the lungs for carbon monoxide corrected; TLV, target lobe volume; 6MWD, 6-min walk distance; SGRQ, St. George's respiratory questionnaire. 
Table 7. qCT results of discordant case B

\begin{tabular}{lcclr}
\hline & Total volume & Emphysema ratio, \% $(-950 \mathrm{HU})$ & Planar perfusion, \% (geometric mean analysis*) \\
\hline RUL & 1,423 & 32.2 & RUZ & 7 \\
RML & 612 & 4.1 & RMZ & 25 \\
RLL & 1,444 & 16.9 & RLZ & 20 \\
LUL & 1,831 & 19.4 & LUZ & 9 \\
& & 21.1 & LMZ & 24 \\
LLL & 1,419 & LLZ & 15 \\
\hline
\end{tabular}

The RUL was chosen as target lobe according to qCT and planar perfusion results. qCT, quantitative computed tomography; HU, Hounsfield units; LLL, left lower lobe; LUL, left upper lobe; RLL, right lower lobe; RML, right middle lobe; RUL, right upper lobe; * Planar perfusion geometric mean analysis in six equal zones; LLZ, left lower zone; LUZ, left upper zone; RUZ, right upper zone; RLZ, right lower zone; RMZ, right middle zone.

this too could have contributed to the lack of sustained effect.

In the second discordant case, $\mathrm{B}$, the right upper lobe was chosen because of the highest emphysema ratio on qCT and the right upper zone had the lowest perfusion on planar quantitation (Table 7). On RAHVQSQ analysis (Table 8), the left lower lobe was a better target with the lowest VQDI, higher heterogeneity, and equivalent volume. In this participant, volume reduction was not achieved and a repeat procedure was not possible due to patient clinical decline and he was lost to follow-up.

\section{Secondary Outcomes}

There was a $76 \%$ mean relative reduction of the target lobe VQDI following EBV-LVR at 3 months, from $0.59 \pm$ 0.28 to $0.14 \pm 0.30$ and a mean difference (MD) of -0.45 (95\% CI $-0.62,-0.29, p<0.0001)$, compatible with good atelectasis response to EBV-LVR. However, there were no statistically significant post EBV-LVR changes to VQDI in the ipsilateral untreated lobe (MD 0.11, $p=$ $0.39)$, the overall contralateral lung (MD 0.02, $p=0.87$ ), the counterpart lobe to the target lobe in the contralateral lung (MD $-0.02, p=0.87$ ), and counterpart lobe to the ipsilateral untreated lobe in the contralateral lung (MD-0.03, $p=0.87)$ following the procedure at 3 months.

There was a significant improvement in diffusing lung capacity (DLCO) at 6 months with a MD of $3.75 \%(p=$ 0.04 ). The improvement was initially not significant at 3 months (MD 2.63\%, $p=0.12$ ).

The association between the change of DLCO and the VQDI of the target lobe and ipsilateral untreated lobe was not identified. Further analyses showed there was an association between the improvement in DLCO and the baseline VQDI of the contralateral lung counterpart lobe
Table 8. RAHVQSQ analysis of discordant case B

\begin{tabular}{lccll}
\hline & Volume, \% & Ventilation, \% & Perfusion, \% & VQDI \\
\hline RUL & 22.7 & 18.80 & 17.98 & 0.66 \\
RML & 10.53 & 17.49 & 17.77 & 2.81 \\
RLL & 16.68 & 9.32 & 15.74 & 0.42 \\
LUL & 27.72 & 42.90 & 31.68 & 1.77 \\
LLL & 20.35 & 11.49 & 16.84 & 0.47 \\
\hline
\end{tabular}

The LLL was chosen as the target lobe which had a lower VQDI than the treated RUL. RUL, right upper lobe; RML, right middle lobe; RLL, right lower lobe; LUL, left upper lobe; LLL, left lower lobe; VQDI, ventilation-perfusion differential index; RAHVQSQ, Royal Adelaide Hospital ventilation perfusion SPECT/CT quantitation.

to the treated lobe $(p=0.024)$. In this counterpart lobe, for every 1 unit VQDI above the mean baseline $(0.98 \pm$ $0.46)$, there was an estimate of $18.5 \%$ increase in DLCO at 3 months post EBV-LVR (Estimate $=18.5,95 \%$ CI 2.42 , 34.67).

\section{Clinical Outcomes and Adverse Events}

Statistically and clinically significant improvements from baseline were seen at both 3 and 6 months for FEV1, total lung capacity, RV, SGRQ score, and target lobe volume (shown in Table 9). The responder threshold for FEV1 was defined as a greater or equal to $100 \mathrm{~mL}$ increment from baseline [8]. There was an absolute mean improvement in FEV1 of $150 \mathrm{~mL}$ at 3 months $(p<0.001)$, with $8 / 16(50 \%)$ participants classified as a responder. At 6 months, the mean improvement was $90 \mathrm{~mL}(p=0.02)$, with $9 / 15(60 \%)$ of the participants still classified as responders. 
Table 9. Effective clinical outcomes

\begin{tabular}{|c|c|c|c|c|c|c|}
\hline FEV1, \%pred & $32.3 \pm 7.95$ & $39.8 \pm 8.00$ & $37.4 \pm 8.40$ & $\begin{array}{l}\text { Pre }-3 \\
\text { Pre }-6\end{array}$ & $\begin{array}{l}6.82(3.85,9.78) \\
4.42(1.38,7.45)\end{array}$ & $\begin{array}{r}<0.0001 \\
0.0058\end{array}$ \\
\hline FVC, L & $2.39 \pm 0.60$ & $3.02 \pm 0.68$ & $2.81 \pm 0.66$ & $\begin{array}{l}\text { Pre }-3 \\
\text { Pre }-6\end{array}$ & $\begin{array}{l}0.57(0.32,0.82) \\
0.36(0.10,0.61)\end{array}$ & $\begin{array}{r}<0.0001 \\
0.0074 \\
\end{array}$ \\
\hline FVC, \%pred & $69.7 \pm 12.7$ & $87.6 \pm 22.4$ & $83.6 \pm 14.9$ & $\begin{array}{l}\text { Pre }-3 \\
\text { Pre }-6\end{array}$ & $\begin{array}{l}16.7(8.96,24.4) \\
11.9(4.02,19.8)\end{array}$ & $\begin{array}{l}0.0001 \\
0.0044\end{array}$ \\
\hline TLC, L & $6.72 \pm 1.11$ & $6.48 \pm 1.01$ & $6.38 \pm 1.19$ & $\begin{array}{l}\text { Pre }-3 \\
\text { Pre }-6\end{array}$ & $\begin{array}{l}-0.34(-0.52,-0.17) \\
-0.24(-0.43,-0.06)\end{array}$ & $\begin{array}{l}0.0004 \\
0.0118\end{array}$ \\
\hline $\mathrm{RV}, \mathrm{L}$ & $4.19 \pm 0.82$ & $3.43 \pm 0.59$ & $3.41 \pm 0.71$ & $\begin{array}{l}\text { Pre }-3 \\
\text { Pre }-6\end{array}$ & $\begin{array}{l}-0.80(-1.12,-0.48) \\
-0.67(-1.01,-0.34)\end{array}$ & $\begin{array}{r}<0.0001 \\
0.0003\end{array}$ \\
\hline RV, \%pred & $201 \pm 37.1$ & $162 \pm 20.4$ & $166 \pm 28.5$ & $\begin{array}{l}\text { Pre }-3 \\
\text { Pre }-6\end{array}$ & $\begin{array}{l}-39.8(-55.8,-23.8) \\
-33.3(-50.1,-16.6)\end{array}$ & $\begin{array}{r}<0.0001 \\
0.0003 \\
\end{array}$ \\
\hline DLCO, \%pred & $38.5 \pm 13.6$ & $41.3 \pm 12.4$ & $44.1 \pm 11.2$ & $\begin{array}{l}\text { Pre }-3 \\
\text { Pre }-6\end{array}$ & $\begin{array}{l}2.63(-0.71,5.98) \\
3.75(0.23,7.27)\end{array}$ & $\begin{array}{l}0.1183 \\
0.0378\end{array}$ \\
\hline 6MWD, m & $336 \pm 113$ & $357 \pm 122$ & $391 \pm 111$ & $\begin{array}{l}\text { Pre }-3 \\
\text { Pre }-6\end{array}$ & $\begin{array}{l}16.0(-25.4,57.5) \\
25.1(-21.7,71.9)\end{array}$ & $\begin{array}{l}0.4332 \\
0.2787\end{array}$ \\
\hline SGRQ, point & $65.1 \pm 11.0$ & $54.6 \pm 14.9$ & $51.3 \pm 20.2$ & $\begin{array}{l}\text { Pre }-3 \\
\text { Pre }-6\end{array}$ & $\begin{array}{l}-10.9(-19.3,-2.46) \\
-14.1(-22.9,-5.34)\end{array}$ & $\begin{array}{l}0.0132 \\
0.0027\end{array}$ \\
\hline
\end{tabular}

BMI, body mass index; FEV1, force expiratory volume in $1 \mathrm{~s}$; FVC, force vital capacity; RV, residual volume; TLC, total lung capacity; DLCOcorr, diffusing capacity of the lungs for carbon monoxide corrected; SGRQ, St. George's respiratory questionnaire. * Analysis was done per protocol, using linear mixed-effects models.

The responder threshold for $\mathrm{RV}$ reduction was defined as greater or equal to $430 \mathrm{~mL}$ [9]. At 3 months, 10/15 $(66 \%)$ participants had a mean reduction in $\mathrm{RV}$ of $800 \mathrm{~mL}$ $(p<0.0001)$. The result was sustained at 6 months with a MD of $670 \mathrm{~mL}(p=0.0003)$.

The responder threshold for target lobe volume reduction was defined as greater or equal to $350 \mathrm{~mL}$ [7]. Fourteen out of $16(88 \%)$ participants were responders, achieving a mean reduction of $973 \mathrm{~mL}(p<0.0001)$ at 3 months.

There was no significant difference in the mean 6MWD; however, $10 / 15(67 \%)$ had a $>26 \mathrm{~m}$ improvement in their $6 \mathrm{MWD}$ at 3 months and 8/11 (73\%) achieved this minimal clinically important difference [10] at 6 months. The absolute and percentage changes from baseline at 3 and 6 months are summarized in Table 9, and the percentage of responders is summarized in Table 10.

The adverse events over 6 months are summarized in Table 11 . There were 4 participants who required valve repositioning and another participant who had repeat bronchoscopy due to mucous plugging. One participant did not achieve atelectasis on follow-up imaging and achieved atelectasis following valve replacement. Two participants required valve replacement a few months post procedure due to partial re-expansion of the treated 
Table 10. MCID responder rate for key clinical outcomes

\begin{tabular}{lrr}
\hline Variable & $\begin{array}{l}3 \text { months, } \\
n(\%)\end{array}$ & $\begin{array}{l}6 \text { months, } \\
n(\%)\end{array}$ \\
\hline FEV1, mL (MCID $\geq 100 \mathrm{~mL})[6]$ & $8 / 16(50)$ & $9 / 15(60)$ \\
RV, mL (MCID $\geq-430 \mathrm{~mL})[7]$ & $10 / 15(63)$ & $10 / 14(71)$ \\
SGRQ, points (MCID $\geq-4)[10]$ & $8 / 16(50)$ & $10 / 15(67)$ \\
6MWD, m (MCID $\geq 26 \mathrm{~m})[9]$ & $10 / 15(67)$ & $8 / 11(72)$ \\
TLVR, mL (MCID $\geq-350 \mathrm{~mL})[8]$ & $14 / 16(88)$ & \multicolumn{1}{c}{ na } \\
\hline
\end{tabular}

FEV1, force expiratory volume in $1 \mathrm{~s}$; RV, residual volume; DLCOcorr, Diffusing capacity of the lungs for carbon monoxide corrected; SGRQ, St. George's respiratory questionnaire; 6MWD, 6-min walk distance; TLVR, target lobe volume reduction; MCID, minimal clinically importance difference.

lobes on subsequent imaging, and they both achieved lobar collapsed following with valve replacement. Another participant had an episode of haemoptysis due to irritation from granulation tissue and subsequently 1 valve was removed and then reinserted 6 weeks post removal. Two procedure-related pneumothoraces occurred in 2 participants (11\%), both occurring within 3 days and both requiring chest tube insertion with subsequent resolution of the pneumothoraces within 7 days. Three respiratory infections were reported including 1 participant who was found to have positive Aspergillus culture on repeat bronchoscopy requiring permanent valve removal before 6 months due to persistent infection and worsening symptoms compared to pre-treatment.

\section{Discussion/Conclusion}

All previous studies $[5,11,12]$ to investigate the role of perfusion scintigraphy in LVR procedures suffered from the same limitation of non-lobar segmentation of lung perfusion, that is, using 2 dimensional planar scans which roughly divides the 2 lungs into 6 equal rectangular zones. The middle zones were excluded for assessment as they included overlapping regions of the upper and lower lobes. Chandra et al. [11] and Argula et al. [12] contend that the target lobe should be the lobe with lowest perfusion.

We believe our study is the first to use VQ SPECT/CT lobar quantitation to determine the target lobe for EBVLVR. Our lobar quantitation technique has refined the way to measure the VQ through anatomically defined lobes. Lobar quantitation with SPECT/CT software is
Table 11. Adverse events during 6 months of follow-up

\begin{tabular}{|c|c|}
\hline Adverse event & $\begin{array}{l}\text { Events } \\
\text { (\% of subject } \\
n=18 \text { ) }\end{array}$ \\
\hline $\begin{array}{l}\text { Pneumothorax (all resolved within } 7 \text { days with } \\
\text { drainage) }\end{array}$ & $2(11)$ \\
\hline COPD exacerbation without pneumonia/infection & $2(11)$ \\
\hline Respiratory infection & $3(17)$ \\
\hline Parainfluenza & 1 \\
\hline Influenza A & 1 \\
\hline Aspergillus* & 1 \\
\hline Haemoptysis ${ }^{\#}$ & $2(11)$ \\
\hline Persistent cough & $1(6)$ \\
\hline EBV reposition & $4(22)$ \\
\hline Loss of valve effect & $2(11)$ \\
\hline No atelectasis & $1(6)$ \\
\hline $\begin{array}{l}\text { Granulation tissue/haemoptysis - temporary } \\
\text { valve removal and reinsertion" }\end{array}$ & $1(6)$ \\
\hline $\begin{array}{l}\text { Permanent EBV removal from chronic Aspergillus } \\
\text { infection and cough* }\end{array}$ & $1(6)$ \\
\hline
\end{tabular}

COPD, chronic obstructive pulmonary disease; EBV, endobronchial valve. * This patient had valve removal from Aspergillus infection. * This patient had haemoptysis from granulation tissue and required temporary valve removal and reinsertion.

currently commercially available. We have independently devised a program with a different approach that we found to be more intuitive and flexible to the operator in terms of delineating the locations of the fissures which we found helpful especially when the lungs are grossly disfigured and the fissures are deficient.

We have undertaken a unique approach to the analysis of results by considering both the $\mathrm{V}$ and the $\mathrm{Q}$ data, with each corrected for lobar volume, to create an index that we have called VQDI. We think the target lobe choice should not be based on perfusion scan alone as it is only half of the evaluation for gas exchange. This is relevant in advanced emphysema and is well demonstrated in a VQ scan whereby the heterogeneity of radioactivity in the ventilation scan can be drastically different from heterogeneity of radioactivity in the perfusion scan. There are often lobes which appear perfused but not ventilated on the scan. To assume such a lobe has equal value for gas exchange as another lobe with equivalent perfusion but significantly higher ventilation would be erroneous.

The main premise of our analysis is that it is important to know the concentration of air and concentration of blood in each lobe to assess the capacity of each lobe to perform gas exchange. Thus, the percentage radioactivity 
of each radiopharmaceutical was divided by the percentage volume. The absolute amount of radioactivity in each lobe can be misleading for target lobe determination because the volumes of the lobes are different. A small healthy lobe may have a lower absolute amount of radioactivity than a large diseased lobe.

In emphysema, there is gas trapping from the loss of lung compliance. The ventilation scan only demonstrates the inspired radioactive gas. The dead space appears photopenic or "cold" on the scan. For analysis of the amount of inspired air that is available for gas exchange, we estimated the "concentration of air" by dividing the percentage radioactivity of the ${ }^{99 \mathrm{~m}} \mathrm{Tc}$ Technegas by the percentage volume of the lobe.

In emphysema, there is shunting of blood away from diseased lobes in preference of the healthier lobes to maximize gas exchange. For analysis of the amount of blood that is available for gas exchange, we estimated the "concentration of blood" by dividing the percentage radioactivity of the ${ }^{99 \mathrm{~m}} \mathrm{Tc}$ macro aggregated albumin by the percentage volume of the lobe.

VQDI is a product of concentration of air and concentration of blood. It compares the degree of emphysema between the lobes by evaluating the combined effects of gas trapping and blood shunting. The worse the emphysema, the lower the index.

In this study, there is evidence of statistically significant subjective and objective improvement in the subjects who received our standard of care that used qCT with the assistance of planar VQ quantitation to determine the target lobe. Thus, qCT is a valid gold standard of imaging for this purpose. A very high concordance between VQDI and $\mathrm{qCT}$ supports the role of VQDI.

The principal aim of this study is verifying the value VQDI as a parameter for target lobe selection in EBVLVR by comparing with the gold standard test of qCT. The intention of this study is not to evaluate VQDI as an alternative replacement for qCT. It is also not the intention of this study to evaluate if VQDI complements qCT. Nevertheless, in our clinical practice, the 2 techniques are complementary. To assess disease burden, qCT evaluates tissue density, whereas VQDI evaluates the combined effects of gas trapping and blood shunting. Applying both techniques adds to the confidence of target lobe choice, especially in homogenous emphysema. As illustrated in the first discordant case where the emphysema ratio was uniform (shown in Table 4) and only the left oblique fissure was intact, only the left lung could be the target for EBV-LVR. However, the RAHVQSQ analysis showed a significant VQDI heterogeneity between the left lower

Use of VQ SPECT to Select Target Lobe for EBV-LVR lobe and the left upper lobe, where the lower lobe had the lower VQDI (Table 5). If this participant was not recruited, the target lobe choice by our panel may have been different. Of note the second discordant case also did not achieve a sustained benefit. The potentially better clinical outcome with target lobe choice based on VQDI for the discordant cases is speculative and is beyond the scope of this observational study.

The effect of EBV on V and Q has been studied recently. The first pilot study by Chung et al. [13] using an Australian cohort of 8 patients underwent left upper lobe EBV treatment. Serial VQ SPECT perfusion scintigraphy was done at baseline, day 30 and 90 . There was a significant reduction of both the $\mathrm{V}$ and $\mathrm{Q}$ in the treated lobe and the total left lung. They also found the right lower lobe and the overall right lung's $\mathrm{V}$ and $\mathrm{Q}$ were increased. The reduction of ventilation in the treated lobe was consistent with the function of EBVs to achieve atelectasis, while reduced perfusion was thought to likely be due to hypoxic vasoconstriction in the same hypoventilated area, resulting in redistribution of perfusion to other lung regions. The likelihood of the left lower lobe (untreated ipsilateral lobe) decrease in perfusion could be due to the "dilution" effect from the compensatory hyperinflation.

Another study by Pizarro and colleagues [14] from Germany also found a significant reduction in target zone perfusion and ventilation of 26 patients at 8 weeks. They found the perfusion in the contralateral lung increased significantly by $5.96 \%$ (relative change, $p=0.005$ ). Pizarro et al. [14] also found an association between the 6MWD responders (69.2\% subject, based on at least $25 \mathrm{~m}$ improvement on $6 \mathrm{MWD}$ ) who had both their perfusion and ventilatory changes reduced in the target lobe.

More recently, Hsu et al. [15] reported the VQ changes and gas transfer capacity corrected for alveolar volume, KCO of 11 patients. There were significant decreases in the $\mathrm{V}$ and $\mathrm{Q}$ activity of the targeted lung and increases in the non-targeted lung at 1 month. The trends were still observed at 12 months, but they were no longer statistically significant. Hsu et al. [15] also reported both the KCO and diffusing gas capacity DLCO improved at 3 months and at 12 months, but not at 1 month. Our study showed a statistically significant improvement in DLCO at 6 months. There was a recorded improvement at 3 months, but the difference did not reach statistical significance.

Using VQDI in our study has further confirmed the observation from reported studies showing significant reduction in the target lobe VQDI 3 months after EBVLVR. However, we did not find a significant difference or 
pattern in the change of VQDI in the ipsilateral untreated lobe, overall contralateral lung, and the counterpart lobes to the treated and untreated lobes of the contralateral lung. There was a delayed improvement in DLCO which became more apparent after 6 months, suggesting a remodelling process following the VQ redistribution to the healthier and better functioning lobes and improvement in the VQ mismatch. Other possible explanations are reduction in the airflow obstruction and improvement of pulmonary hypertension.

In our study, we found an association between the baseline VQDI of the counterpart lobe to treated lobe in the contralateral lung and the DLCO. The higher the baseline VQDI the higher the likelihood of improvement in post EBV-LVR DLCO. This may be explained by an improvement in overall gas exchange in subjects with a healthier contralateral counterpart lobe.

There are several limitations of this study that should be considered. The study design was an observational study without a control group and a small sample size. The target lobe was chosen by the same nuclear medicine physician based on VQ SPECT, although this allows for consistent interpretation by having a reference nuclear physician experienced with the technique. The previous study by El-Barhoun et al. [16] has shown its reproducibility between and within 3 observers with high interand intra-observer concordance. Given the increased radiation exposure with VQ SPECT and CT chest, our study limited the repeated scans only at 3 months; the redistribution of VQ to the other regions of the lungs may be more apparent before or after 3 months. Future studies may be able to repeat the VQ scan at a later time frame that may provide additional scientific value.

In conclusion, our study has validated the value of VQDI derived from VQ SPECT/CT lobar quantitation by comparing it to the gold standard imaging of qCT for target lobe selection in EBV-LVR. In our practice, this functional imaging technique has a complementary role to qCT and adds confidence in target lobe choice. This is especially the case when qCT indicates homogenous emphysema with no clear lobar target. Future prospective randomized study is needed to evaluate the complementary role of the 2 imaging techniques.

\section{Acknowledgements}

We would like to thank Ms. Suzanne Edwards of the Data Management and Analysis Centre, Faculty of Health Sciences, University of Adelaide for providing the statistical analysis of our data. We acknowledge the contribution of our nuclear medicine tech-

nologists Mr. Stirling Ha and Ms. Alice Burke for their preliminary processing of the VQ SPECT/CT data in preparation for analysis by the nuclear physician.

\section{Statement of Ethics}

The study participants were provided written informed consent. The study protocol was approved by the Royal Adelaide Hospital Human Research Ethics/Central Adelaide Local Health Network Governance (HREC/16/RAH/237 R20160624) and the Calvary Health Care Adelaide Human Research and Ethics Committee (16-CHREC-E007).

\section{Conflict of Interest Statement}

V.T., H.J., B.C., B.L., and C.C. have no conflicts of interest to declare. P.N. reports personal fees from Pulmonx and Olympus outside the submitted work. D.G. reports grants and personal fees from Pulmonx outside the submitted work.

\section{Funding Sources}

No funding was received.

\section{Author Contributions}

Authorship and contributorship: V.T., P.N., H.J., D.G., B.C., B.L. and C.C.; conception and design: V.T., P.N., H.J., D.G., B.C., B.L., and C.C.; drafting of the manuscript: V.T., P.N., and C.C.; acquisition and analysis of the data: V.T., P.N., H.J., D.G., B.C., B.L., and C.C.; analysis and interpretation of the data: V.T., P.N., H.J., D.G., and C.C.; drafting of the manuscript for important intellectual content: V.T., P.N., H.J., D.G., B.L., and C.C. All co-authors critically revised the article and gave final approval for this version to be published.

References

Respiration 2021;100:886-897 DOI: $10.1159 / 000515336$
Tee/Nguyen/Jersmann/Grosser/Crouch/ Lorraine/Chew
1 Sciurba FC, Ernst A, Herth FJ, Strange C, Criner GJ, Marquette $\mathrm{CH}$, et al. A randomized study of endobronchial valves for advanced emphysema. N Engl J Med. 2010 Sep 23; 363(13):1233-44.

2 Klooster K, ten Hacken NH, Hartman JE, Kerstjens HA, van Rikxoort EM, Slebos DJ. Endobronchial valves for emphysema without interlobar collateral ventilation. N Engl J Med. 2015 Dec 10;373(24):2325-35.

3 Valipour A, Shah PL, Gesierich W, Eberhardt R, Snell G, Strange C, et al. Patterns of emphysema heterogeneity. Respiration. 2015;90(5): 402-11. 
4 Valipour A, Slebos DJ, Herth F, Darwiche K, Wagner M, Ficker JH, et al. Endobronchial valve therapy in patients with homogeneous emphysema. Results from the IMPACT study. Am J Respir Crit Care Med. 2016 Nov 1;194(9):1073-82.

5 Thomsen C, Theilig D, Herzog D, Poellinger A, Doellinger F, Schreiter N, et al. Lung perfusion and emphysema distribution affect the outcome of endobronchial valve therapy. Int J Chron Obstruct Pulmon Dis. 2016;11:124559.

6 Slebos DJ, Shah PL, Herth FJ, Valipour A. Endobronchial valves for endoscopic lung volume reduction: best practice recommendations from expert panel on endoscopic lung volume reduction. Respiration. 2017;93(2): $138-50$.

7 Herth FJ, Noppen M, Valipour A, Leroy S, Vergnon JM, Ficker JH, et al. Efficacy predictors of lung volume reduction with Zephyr valves in a European cohort. Eur Respir J. 2012 Jun;39(6):1334-42.
8 Donohue JF. Minimal clinically important differences in COPD lung function. COPD. 2005 Mar;2(1):111-24.

9 Hartman JE, Ten Hacken NH, Klooster K, Boezen HM, de Greef MH, Slebos DJ. The minimal important difference for residual volume in patients with severe emphysema. Eur Respir J. 2012 Nov;40(5):1137-41.

10 Puhan MA, Chandra D, Mosenifar Z, Ries A, Make B, Hansel NN, et al. The minimal important difference of exercise tests in severe COPD. Eur Respir J. 2011 Apr;37(4):784-90.

11 Chandra D, Lipson DA, Hoffman EA, Hansen-Flaschen J, Sciurba FC, Decamp MM, et al. Perfusion scintigraphy and patient selection for lung volume reduction surgery. Am J Respir Crit Care Med. 2010 Oct;182(7):93746.

12 Argula RG, Strange C, Ramakrishnan V, Goldin J. Baseline regional perfusion impacts exercise response to endobronchial valve therapy in advanced pulmonary emphysema. Chest. 2013 Nov;144(5):1578-86.
13 Chung SC, Peters MJ, Chen S, Emmett L, Ing AJ. Effect of unilateral endobronchial valve insertion on pulmonary ventilation and perfusion: a pilot study. Respirology. 2010 Oct; 15(7):1079-83.

14 Pizarro C, Ahmadzadehfar H, Essler M, Tuleta I, Fimmers R, Nickenig G, et al. Effect of endobronchial valve therapy on pulmonary perfusion and ventilation distribution. PLoS One. 2015;10(3):e 0118976.

15 Hsu K, Williamson JP, Peters MJ, Ing AJ. Endoscopic lung volume reduction in COPD: improvements in gas transfer capacity are associated with improvements in ventilation and perfusion matching. J Bronchology Interv Pulmonol. 2018 Jan;25(1):48-53.

16 El-Barhoun EN, Chew G, Crouch B, Chatterton B. Reproducibility of a semi-quantitative lobar pulmonary ventilation and perfusion technique using SPET and CT. Hell J Nucl Med. 2017 Jan-Apr;20(1):71-5. 